\title{
Psychological Distress, Fear and Coping Strategies among Hong Kong People During the COVID-19 Pandemic
}

\author{
Sek Ying Chair ${ }^{1}$ - Wai Tong Chien ${ }^{1}$ (D) . Ting Liu ${ }^{1} \cdot$ Louisa Lam $^{2,3} \cdot$ Wendy Cross $^{2} \cdot$ Biswajit Banik $^{2}$. \\ Muhammad Aziz Rahman ${ }^{2,4}$
}

Accepted: 26 September 2021 / Published online: 20 October 2021

(c) The Author(s), under exclusive licence to Springer Science+Business Media, LLC, part of Springer Nature 2021

\begin{abstract}
The COVID-19 pandemic contributed to potential adverse effects on the mental health status of a wide range of people. This study aimed to identify factors associated with psychological distress, fear and coping strategies during the COVID19 pandemic in Hong Kong. A cross-sectional online survey was conducted among general population in Hong Kong. Psychological distress was assessed using the Kessler Psychological Distress Scale; level of fear was evaluated using the Fear of COVID-19 scale; and coping strategies were assessed using the Brief Resilient Coping Scale. Multivariable logistic regression was used to identify key factors associated with these mental health variables. Of the 555 participants, $53.9 \%$ experienced moderate to very high levels of psychological distress, $31.2 \%$ experienced a high level of fear of COVID-19, and $58.6 \%$ showed moderate to high resilient coping. Multivariable logistic regression indicated that living with family members, current alcohol consumption, and higher level of fear were associated with higher levels of psychological distress; perceived stress due to a change in employment condition, being a frontline worker, experiencing 'moderate to very high' distress, and healthcare service use to overcome the COVID-19 related stress in past 6 months were associated with a higher level of fear; and perceived better mental health status was associated with a moderate to high resilient coping. This study identified key factors associated with distress, fear and coping strategies during the pandemic in Hong Kong. Mental health support strategies should be provided continuously to prevent the mental impact of the pandemic from turning into long-term illness.
\end{abstract}

Keywords COVID-19 $\cdot$ Cross-sectional study $\cdot$ Psychological distress $\cdot$ Fear $\cdot$ Coping

This article is part of Topical Collection on Global Psychological Perspective on the COVID-19 Pandemic

Wai Tong Chien

wtchien@cuhk.edu.hk

1 The Nethersole School of Nursing, Faculty of Medicine, The Chinese University of Hong Kong, Room 725A, Esther Lee Building, Shatin, NT, Hong Kong

2 School of Health, Federation University Australia, Berwick, VIC, Australia

3 School of Public Health and Preventive Medicine, Monash University, Melbourne, VIC, Australia

4 Australian Institute of Primary Care and Ageing, La Trobe University, Melbourne, VIC, Australia

\section{Introduction}

The coronavirus disease (COVID-19), caused by a novel strain of coronavirus, was declared a pandemic by World Health Organization (WHO) on 11 March 2020 (World Health Organization, 2020). Centre for Health Protection Department of Health The Government of the Hong Kong Special Administrative Region (2021) stated that the most common incubation period of COVID-19 was around five days, and the case fatality ratio was higher among the older aged population. On 18 June 2021, there were more than 177 million cases and 3.8 million deaths globally (World Health Organization 2021). In Hong Kong, there were 11,885 confirmed cases with 210 deaths (The Government of the Hong Kong Special Administrative Region 2021).

The resultant effects of this pandemic were stressful for the general population in Hong Kong and globally. To limit the spread of COVID-19, in Hong Kong, social distancing was practiced by keeping $\geq 1 \mathrm{~m}$ of distance between persons. 
Other protective measures were implemented such as wearing surgical masks, limiting seating capacity at eateries, temporary closure of high risk premises, working from home, and suspension of school classes (Centre for Health Protection, 2021; Fong, et al., 2020a, b; The Government of the Hong Kong Special Administrative Region, 2020). Transmission control efforts were implemented rapidly and were relatively successful in slowing the spread of COVID19 infection (Kwok et al., 2020). Nevertheless, increased psychological distress among the public adversely affected aspects of life such as school, work and family activities (Brooks et al., 2020; Kwok et al., 2020). Reduced outdoor activities, loss of usual routine, and increased use of electronic devices during the pandemic were positively associated with the development of depressive symptoms (Brooks et al., 2020; Fong, et al., 2020a, b). The increased isolation resulting from social restriction measures has also significantly contributed to social and emotional loneliness, depression, anxiety and insomnia in all walks of life (Tull et al., 2020; Wong et al., 2020).

Furthermore, the ongoing, prolonged COVID-19 pandemic is taking a toll on the global economy, causing a detrimental financial impact (Ashraf, 2020; Sansa, 2020). Census and Statistics Department, The Government of the Hong Kong Special Administrative Region (2021a) announced a $7 \%$ seasonally adjusted unemployment rate between November 2020 and January 2021, which was the highest for the past 17 years. Unemployment has resulted in not only loss of income but limitations of capacity of people to plan ahead. This would subsequently increase stress levels and reduced self-esteem and general well-being of general population globally (Voßemer et al., 2018; Yao \& Wu, 2021).

Compared with general population, healthcare workers face more mental health challenges as they may have direct contact with infected patients (Gan et al., 2020; Lu et al., 2020; Pappa et al., 2020). Fear of spreading COVID-19, stigmatization by family and friends, and increased workload are significant factors related to the adverse psychological outcomes among healthcare workers (Cai et al., 2020; Lu et al., 2020; Magill et al., 2020). A systematic review of the prevalence of psychological distress amongst frontline healthcare workers caring for COVID-19 patients reported the prevalence of depression, anxiety and stress among frontline healthcare workers as $24 \%, 26 \%$ and $45 \%$ respectively (Salari et al., 2020).

In Hong Kong, recent population-based studies examined the impacts of the COVID-19 pandemic on mental health (Choi et al., 2020; Tso \& Park, 2020; Zhao et al., 2020). They reported that more than half of the respondents experienced worsened mental health, including clinical levels of anxiety, depression and/or stress (Choi et al., 2020; Tso \& Park, 2020). Compared to the public mental health surveys in 2016 and 2017, Zhao et al. (2020) reported an alarming twofold increase in psychological distress (stress, anxiety and depression) and unhappiness among Hong Kong adults during the COVID-19 pandemic period. However, research evidence examining the factors associated with psychological distress, fear of COVID-19 and coping strategies in Hong Kong is limited. Recent studies have suggested that being female and young, lacking local medical resources and inefficiencies within the public health system are associated with a higher level of psychological distress among the Chinese populations (Liu et al., 2020; Qiu et al., 2020). Lack of COVID-19 information, fear of contracting COVID-19, insufficient personal protective equipment and social distancing measures were also associated with a higher risk of anxiety among the public in Hong Kong (Choi et al., 2020; Yeung et al., 2020). To cope with the pandemic, problemfocused coping, seeking social support, avoidance of the situation (e.g. distracting oneself, denying the significance of the situation), and positive appraisal of the current crisis could be the most common coping responses, as suggested in a narrative synthesis of coping related survey data in general population (Chew et al., 2020).

Research evidence exploring factors associated with psychological distress, fear of COVID-19 and coping strategies amongst the general population such as community members, healthcare workers and health care service users in Hong Kong and worldwide is scarce. Since the pandemic shows no sign of ending, understanding the pandemic's impacts on mental health and coping strategies amongst the public, and identifying their predictors, are essential to design psychological support strategies during/after the pandemic, and to prevent long-term mental health problems. Therefore, this study aimed to assess the levels of psychological distress, fear of the COVID-19 infection and main coping strategies used among a wide range of people in Hong Kong, and to identify key factors associated with these mental health conditions/variables.

\section{Methods}

\section{Study Design and Participants}

A cross-sectional survey study was conducted using an online platform. The survey link was distributed via social media, text messages, emails and word of mouth to reach the general population in Hong Kong. The study population included Hong Kong residents who were aged $\geq 18$ years and able to respond to an online questionnaire in English. Participants, including the general public, healthcare workers, health care service users, and university students and staff, were recruited from various community settings and groups via the online platform and social media between December 2020 and mid-January 2021. 


\section{Sample Size Estimation}

The sample size was calculated by OpenEpi. Considering $7,428,300$ as the population of Hong Kong at the end of 2020 (Census and Statistics Department, The Government of the Hong Kong Special Administrative Region, 2021b), the prevalence of worsened mental health among Hong Kong residents during the COVID-19 pandemic ranging from $25.4 \%$ to $65.6 \%$ (Choi et al., 2020; Tso \& Park, 2020), at $95 \%$ confidence intervals and $80 \%$ power, the estimated minimum sample size was 292. Snowball sampling of friends, university staff and students, and invitation messages in social media were used to recruit participants.

\section{Study Instruments}

A structured online survey questionnaire was adopted from an international study led by researchers in Australia (Rahman et al., 2020). Participants first completed a series of questions about socio-demographic information such as age, gender, education level, and employment, and other information related to any perceived stress due to change in employment, being a frontline worker, change in financial situation during the pandemic, and patterns of unhealthy lifestyle (smoking and drinking).

Psychological distress was assessed using the 10-item Kessler Psychological Distress Scale (K10) (Furukawa et al., 2003). The $\mathrm{K} 10$ is a reliable and valid scale, with Cronbach's alpha of 0.92 (Rahman et al., 2021). The Cronbach's alpha of K10 was 0.95 in this study. The K10 items were rated on a 5 -point Likert scale $(1=$ none to $5=$ all the time $)$, with the possible total score range from 10 to 50 . Higher scores indicated higher level of psychological distress.

The level of fear of COVID-19 was evaluated using the 7-item Fear of COVID-19 scale (FCV-19S) (Ahorsu et al., 2020). FCV-19S is a reliable and valid scale, with Cronbach's alpha of 0.82-0.87 (Ahorsu et al., 2020; Rahman et al., 2021). The Cronbach's alpha of FCV-19S in this study was 0.91 . Responses also used a 5-point Likert scale $(1=$ strongly disagree to $5=$ strongly agree). The total score ranged from 7 to 35 . Higher scores indicated higher level of fear of COVID-19.

Coping strategies were assessed using the 4-item Brief Resilient Coping Scale (BRCS) (Sinclair \& Wallston, 2004). BRCS has been widely used during the COVID-19 pandemic with acceptable psychometric properties (Cronbach's alpha ranged from 0.63 to 0.79) (López et al., 2020; Rahman et al., 2021). In this study, Cronbach's alpha was 0.88. It was also assessed with a 5-point Likert scale ranging from 1 (nothing) to 5 (a lot). Responses were summed to create a total score (range $=4-20$ ), with higher scores signifying a higher level of resilient coping.

\section{Data Collection}

Ethics approval was obtained from the Survey and Behavioural Research Ethics Committee of The University (SBRE20-172). After obtaining the ethics approval, an invitation with the information and instructions of the online survey, together with its hyperlink (webpage) and QR code, were shared through different social media platforms (e.g., Facebook, Instagram, LinkedIn, and Twitter), emails and text messages. The plain language information statement and consent form, which provided a thorough explanation of the study including the research aims, procedure, risks and benefits, and voluntary participation, appeared on the first screen-page of the online survey. Only those who provided consent on the first page and admitted to be an adult (aged 18 or above) on the second page, by ticking the button (Yes) at the end of the page(s), would confirm their agreement and their eligibility of participation in this study, respectively. After that, the subsequent webpages contained the full set of study questionnaires for individual participants to complete. All items on each page should be completed before participants could move to the next page. It took 15 to 20 min to complete the survey; and all of their responses were anonymous. No personal identity and information of the participants such as name, residential address and identification number were collected.

\section{Data Analysis}

Data entries and statistical analyses were performed by using IBM SPSS 25.0 (IBM Crop., Armonk, NY). Descriptive statistics, such as mean and standard deviation and frequency and percentage, were used to summarize and present the data of participants' sociodemographic characteristics and study variables. To assess mental health conditions and to be consistent with the previous study (Rahman et al., 2020), participants' psychological distress, fear of the COVID infection and coping strategies were grouped into different levels. Psychological distress (K10 score) was defined into low (score 10-15), moderate (score 16-21), high (score 22-29) and very high (score 30-50); fear of COVID-19 (FCV-19S score) was defined into low (score 7-21) and high (score 22-35); and coping strategies (BRCS score) were categories into low (score 4-13) and moderate to high (score 14-20).

Univariate logistic regression analyses were conducted to explore the association between variables. Those factors with $p<0.25$ were selected as potential confounding variables for multivariate logistic regression to delineate factors associated with the main study variables (psychological distress, fear of COVID-19 and coping strategies). Results of the multivariate logistic regression models for each of those three variables were calculated and presented with adjusted odds ratios (AORs) and $95 \%$ confidence intervals 
(CIs). Level of significance of all statistical tests used was set at 0.05 (two-sided).

\section{Ethical Considerations}

Ethics approval was obtained from the Survey and Behavioural Research Ethics Committee at The Chinese University of Hong Kong (No. SBRE-20-172). The study was conducted following the principles stated in the Declaration of Helsinki. No personal identity and information was collected or reported. Informed consent for participation in the study was obtained on the first page of the survey where study information was provided prior to participants completing the questionnaire.

\section{Results}

\section{Characteristics of Participants}

In total, 555 individuals completed the survey. Characteristics of the participants are listed in Table 1. The mean age of the participants was 47.7 years $( \pm 12.89)$ and $67.2 \%$ were female. The majority of the participants were living with their family members (85.4\%) and had a bachelor's degree or above (80.7\%). Over one-third of the participants $(40.7 \%)$ identified themselves as frontline or essential service workers during the COVID-19 pandemic. For mental health measures, K10 scores ranged from 10-40 $(M=18.8$; $S D=7.38)$; FCV-19S scores ranged from 7-33 $(M=17.78$; $S D=6.70$; and BRCS scores ranged from 4-20 $(M=13.88$, $S D=3.17)$. Of the 555 participants, more than half (53.9\%) experienced moderate to very high levels of psychological distress, one-third (31.2\%) experienced a high level of fear of COVID-19, and over half (58.6\%) showed moderate to high resilient coping (Supplementary Tables 1, 2 and 3, respectively).

\section{Psychological Distress}

The univariate analyses (Table 2) showed significant associations between moderate to very high level of psychological distress and other variables. After adjusting for potential confounding variables, the multivariate logistic regression test (Table 2) indicated some factors associated with psychological distress. Living with family members $(\mathrm{p}=0.010$, AOR: 3.24, 95\% CIs: 1.33-7.94), current alcohol consumption ( $\mathrm{p}=0.034$, AOR: $2.07,95 \%$ CIs: $1.06-4.05)$, and high level of fear of COVID-19 ( $<<0.001$, AOR: 5.76, 95\% CIs: 2.76-12.15) were associated with 'moderate to very high' psychological distress of the participants. Conversely, participants aged $30-59$ years $(\mathrm{p}=0.031$, AOR: $0.30,95 \%$ CIs: $0.10-0.90)$ and $\geq 60$ years ( $\mathrm{p}=0.001$, AOR: $0.13,95 \%$ CIs:
0.04-0.45), perceived good to excellent mental health status ( $\mathrm{p}<0.001$, AOR: 0.13 , 95\% CIs: 0.06-0.26), and 'moderate to high' resilient coping ( $\mathrm{p}=0.028$, AOR: $0.51,95 \%$ CIs: $0.28-0.93$ ) were associated with low levels of psychological distress.

\section{Fear of COVID-19}

Table 3 showed the univariate analyses to identify associations between high level of fear of COVID-19 and other variables. The multivariate logistic regression results (Table 3) showed that perceived moderate to a great deal of stress due to a change in employment conditions $(p=0.002$, AOR:4.12, 95\%CIs: 1.72-9.88), being a frontline or essential service worker ( $p=0.017$, AOR: $2.72,95 \%$ CIs: $1.19-6.19$ ), experiencing 'moderate to very high' psychological distress ( $p<0.001$, AOR: 6.00, 95\%CIs: 2.84-12.70), and using healthcare services to overcome COVID-19 related stress in past 6 months ( $p=0.002$, AOR:6.38, 95\%CI: 1.98-20.55), were associated with a high level of fear of the COVID-19 infection. However, a low level of fear of the COVID-19 infection was related to being a nurse $(p=0.032$, AOR:0.38, 95\%CIs: 0.16-0.92).

\section{Coping Strategies}

The univariate analyses identified a number of variables were associated with moderate to high level of resilient coping (Table 4). After adjusting for potential confounding variables, as shown in Table 4, participants who perceived good to excellent mental health status during the COVID-19 pandemic ( $p=0.001$, AOR:2.76, 95\%CIs:1.53-4.95) were more likely to have 'moderate to high' resilient coping. On the contrary, those who perceived a change due to an unsure financial situation ( $\mathrm{p}=0.015$, AOR:0.43, 95\% CIs: $0.22-0.85)$, being tested positive ( $\mathrm{p}=0.035$, AOR:0.10, 95\%CIs: $0.01-0.85$ ) or negative on the COVID-19 screening test ( $\mathrm{p}=0.024$, AOR:0.42, 95\%CIs: $0.20-0.89)$, high levels of fear of the COVID-19 infection ( $\mathrm{p}=0.029$, AOR:0.51, 95\%CIs: $0.28-0.93$ ), and using healthcare services to overcome the COVID-19 related stress in past 6 months $(p=0.050$, AOR:0.36, 95\%CI: 0.13-1.00) were associated with a low level of resilient coping.

\section{Discussion}

Our findings showed over half (54\%) of the participants experienced 'moderate to very high' levels of psychological distress and about one-third (31\%) reported a high level of fear of the COVID-19 infection. Despite having moderate to high levels of psychological distress and fear, more than half (60\%) demonstrated 'moderate to high' resilient coping. Our 
Table 1 Characteristics of the study population

\begin{tabular}{ll}
\hline Characteristics & $\begin{array}{l}\text { Total } \\
\mathrm{n}(\%)\end{array}$ \\
\hline Total study participants & 555 \\
Age (in years) & $\mathbf{5 5 3}$ \\
Mean $( \pm \mathrm{SD})$ & $47.7(12.89)$ \\
Range & $19-76$ \\
Age groups & $\mathbf{5 5 3}$ \\
$18-29$ years & $51(9.2)$ \\
$30-59$ years & $378(68.4)$ \\
$\geq 60$ years & $124(22.4)$ \\
Gender & $\mathbf{5 5 1}$
\end{tabular}

$181(32.8)$

Female

$370(67.2)$

Born in Hong Kong

Yes

$498(90.4)$

No

$53(9.6)$

Living status

549

Live without family members (on your own/shared house/others)

$80(14.6)$

Live with family members

$469(85.4)$

Highest educational/vocational qualification

549

Secondary/Higher secondary/Intermediate/Grade 7-12

$13(2.4)$

Certificate/Diploma/Trade qualification

$93(16.9)$

Bachelor/Master/PhD

$443(80.7)$

Current employment condition

550

Jobs affected by COVID-19 (lost job/working hours reduced/ afraid of job loss)

Have an income source (employed/Government benefits)

$175(31.8)$

Perceived stress due to change of employment condition

535

A little to none

$419(78.3)$

Moderate to a great deal

$116(21.7)$

Improved working situation due to change of employment

534

A little or none

$497(93.1)$

Moderate to a great deal

37 (6.9)

Self-identification as a frontline or essential service worker

555

No

$329(59.3)$

Yes

$226(40.7)$

Self-identification as healthcare worker

555

No

$273(49.2)$

Yes, doctor

$3(0.5)$

Yes, nurse

$236(42.5)$

Yes, other healthcare worker

43 (7.7)

COVID-19 impacted financial situation

$\mathbf{5 5 5}$

No impact

$420(75.7)$

Yes, impacted positively

$28(5.0)$

No, impacted negatively

$107(19.3)$

Affected by the change in financial situation

544

Not at all

$292(53.7)$

Unsure at this time

85 (15.6)

Somewhat

$130(23.9)$

A great extent

$37(6.8)$

Co-morbidities

554

No

$336(60.6)$

Psychiatric/Mental health issues
$4(0.7)$ 
Table 1 (continued)

\begin{tabular}{|c|c|}
\hline Characteristics & $\begin{array}{l}\text { Total } \\
\mathrm{n}(\%)\end{array}$ \\
\hline Other co-morbidity & 214 (38.6) \\
\hline Co-morbidities & 554 \\
\hline No & $336(60.6)$ \\
\hline Single co-morbidity & $119(21.5)$ \\
\hline Multi co-morbidities & $99(17.9)$ \\
\hline Smoking & 555 \\
\hline Never smoker & $498(89.7)$ \\
\hline Ever smoker (Daily/Non-daily/Ex) & $57(10.3)$ \\
\hline Increased smoking over the last 6 months & 26 \\
\hline No & $7(26.9)$ \\
\hline Yes & $19(73.1)$ \\
\hline Current alcohol drinking & 552 \\
\hline No & $350(63.4)$ \\
\hline Yes & $202(36.6)$ \\
\hline Increased alcohol drinking over the last 6 months & 202 \\
\hline No & $108(53.5)$ \\
\hline Yes & $94(46.5)$ \\
\hline Contact with known/suspected cases of COVID-19 & 546 \\
\hline No & $412(75.5)$ \\
\hline Unsure & $85(15.6)$ \\
\hline Yes, had indirect contact & $37(6.8)$ \\
\hline Yes, provided direct care & $12(2.2)$ \\
\hline Experience related to COVID-19 pandemic & 546 \\
\hline No known exposure to COVID-19 & $452(82.8)$ \\
\hline Tested positive for COVID-19 & $7(1.3)$ \\
\hline Tested negative for COVID-19 but self-isolating & $74(13.6)$ \\
\hline Had recent overseas travel history and was in quarantine & $13(2.4)$ \\
\hline Self-identification as a patient (utilised any health care services) in the last 6 months & 546 \\
\hline No & $344(63.0)$ \\
\hline Yes & $202(37.0)$ \\
\hline Healthcare service use in the last 6 months & 191 \\
\hline Visited a healthcare provider in person & $178(93.2)$ \\
\hline Telehealth consultation/Used helpline & $7(3.7)$ \\
\hline Use both services & $6(3.1)$ \\
\hline Perceived mental health status & 555 \\
\hline Poor to fair & $195(35.1)$ \\
\hline Good to excellent & $360(64.9)$ \\
\hline Healthcare service use to overcome COVID-19 related stress in the last 6 months & 551 \\
\hline No & $480(87.1)$ \\
\hline Yes & $71(12.9)$ \\
\hline Type of healthcare service used to overcome COVID-19 related stress in the last 6 months & 71 \\
\hline Consulted GP & $39(54.9)$ \\
\hline Consulted a Psychologist & $4(5.6)$ \\
\hline Used mental health resources & $6(8.5)$ \\
\hline Used mental health resources available through media & $3(4.2)$ \\
\hline Used mental health support services & $1(1.4)$ \\
\hline Used combination of services & $18(25.4)$ \\
\hline GP: general practitioner; SD: standard deviation & \\
\hline
\end{tabular}




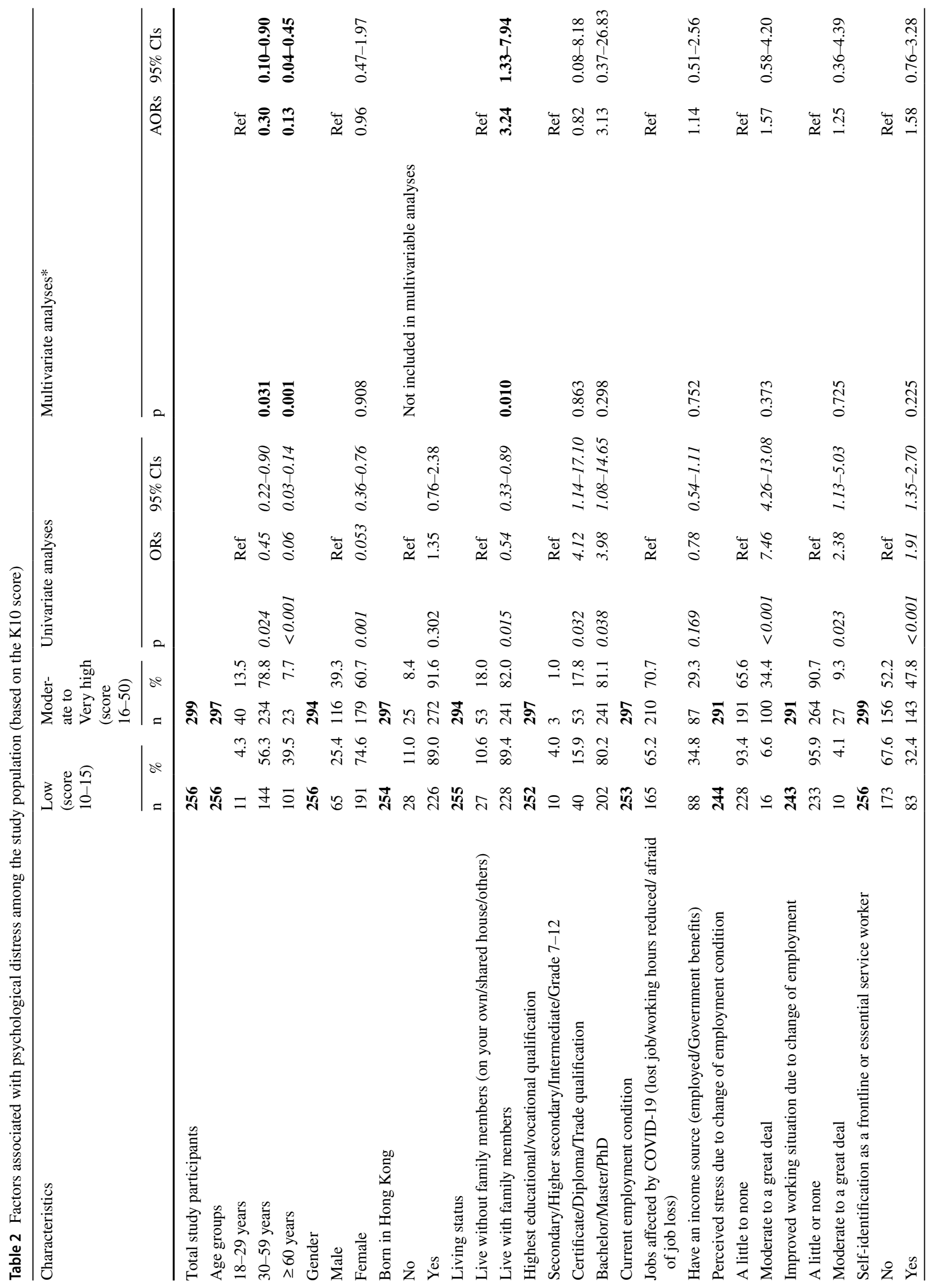




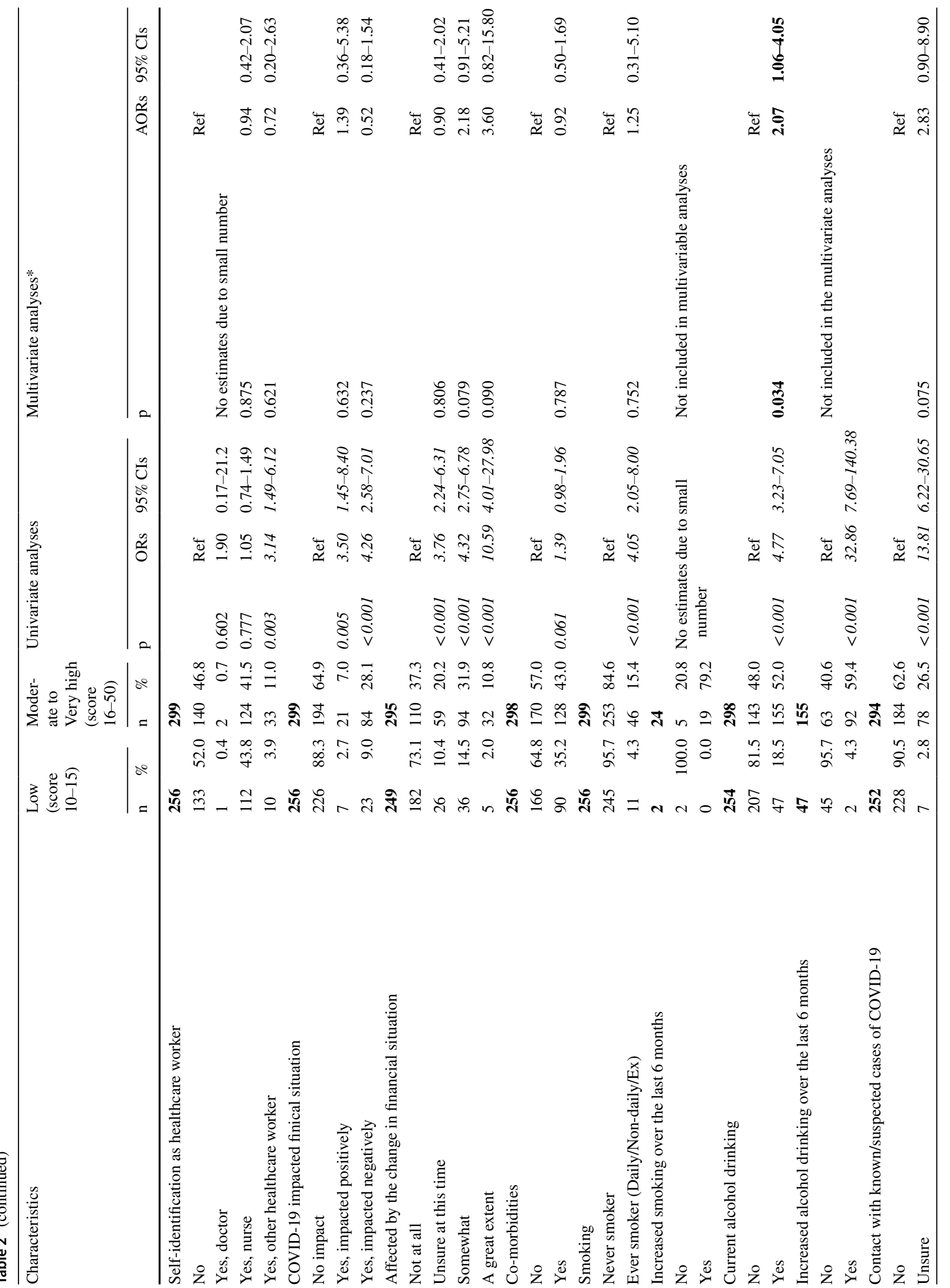




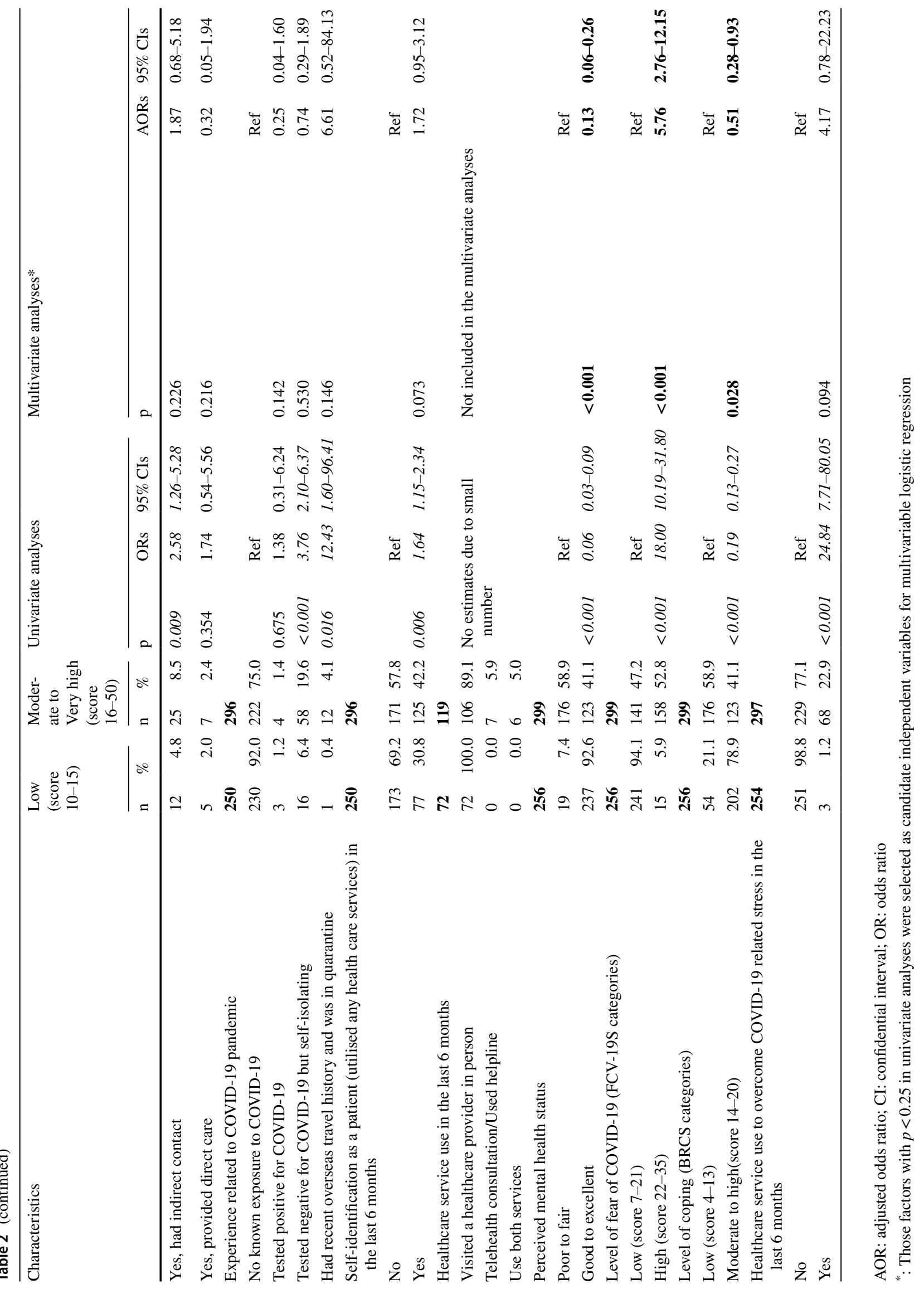




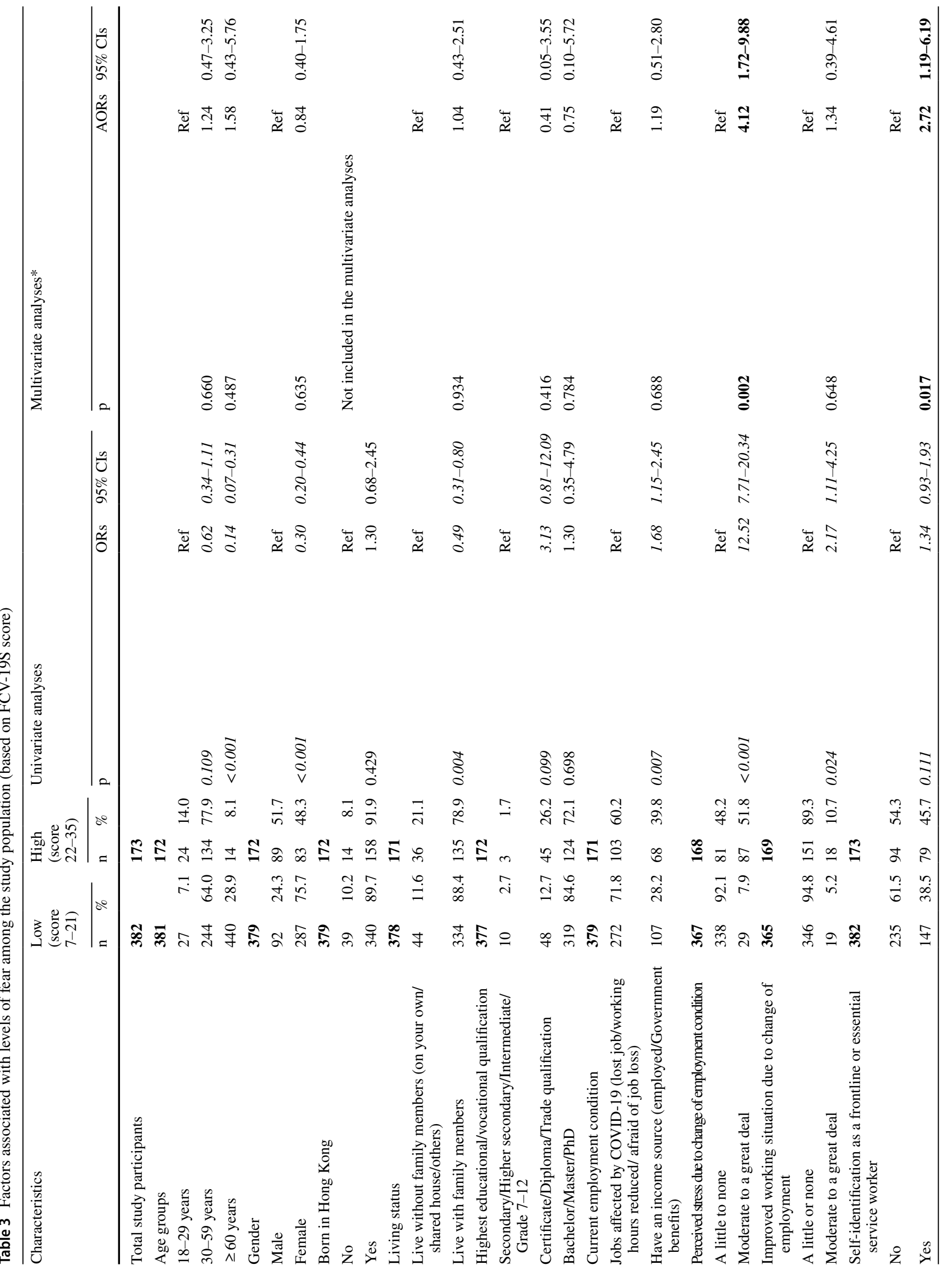




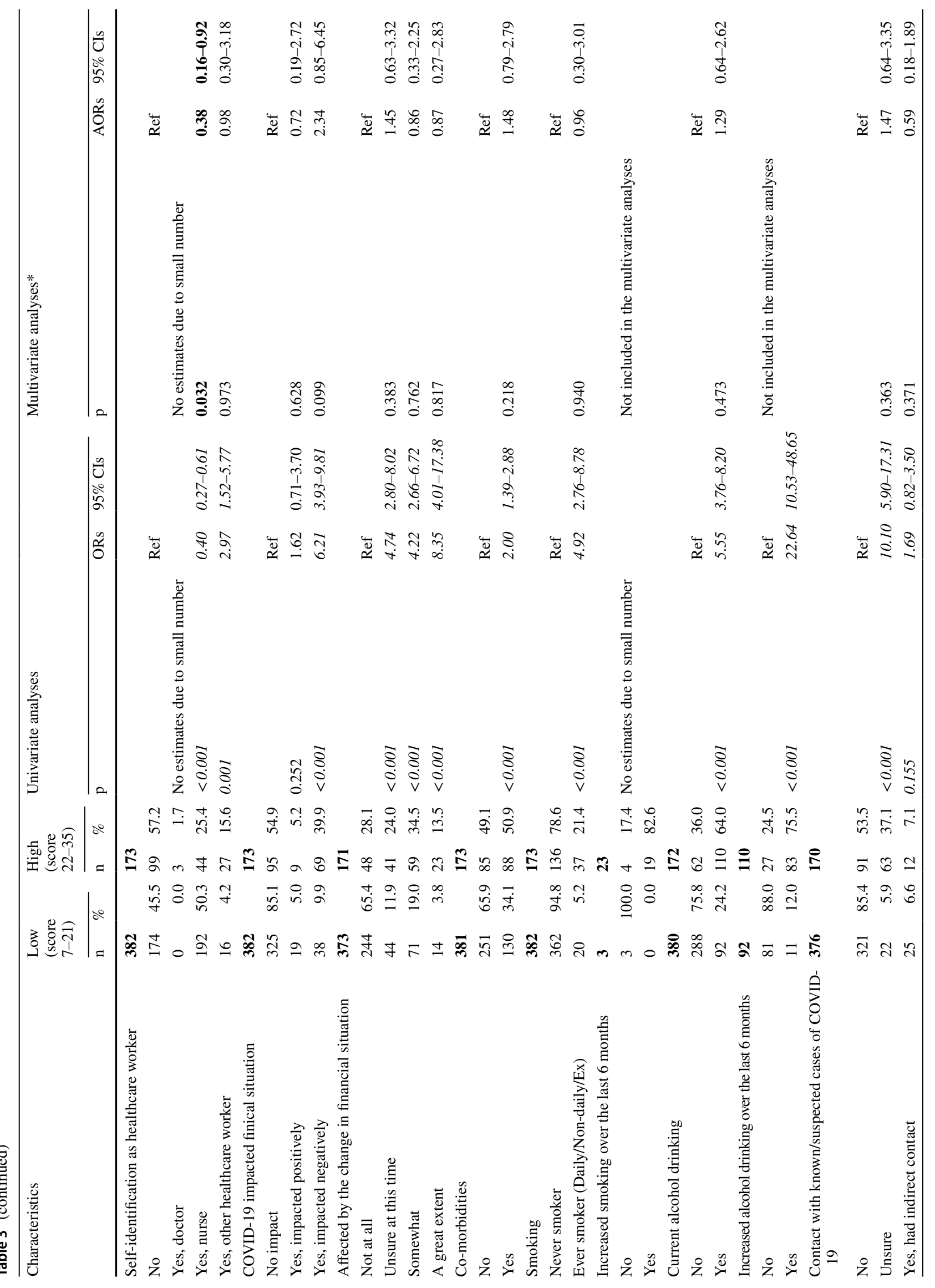




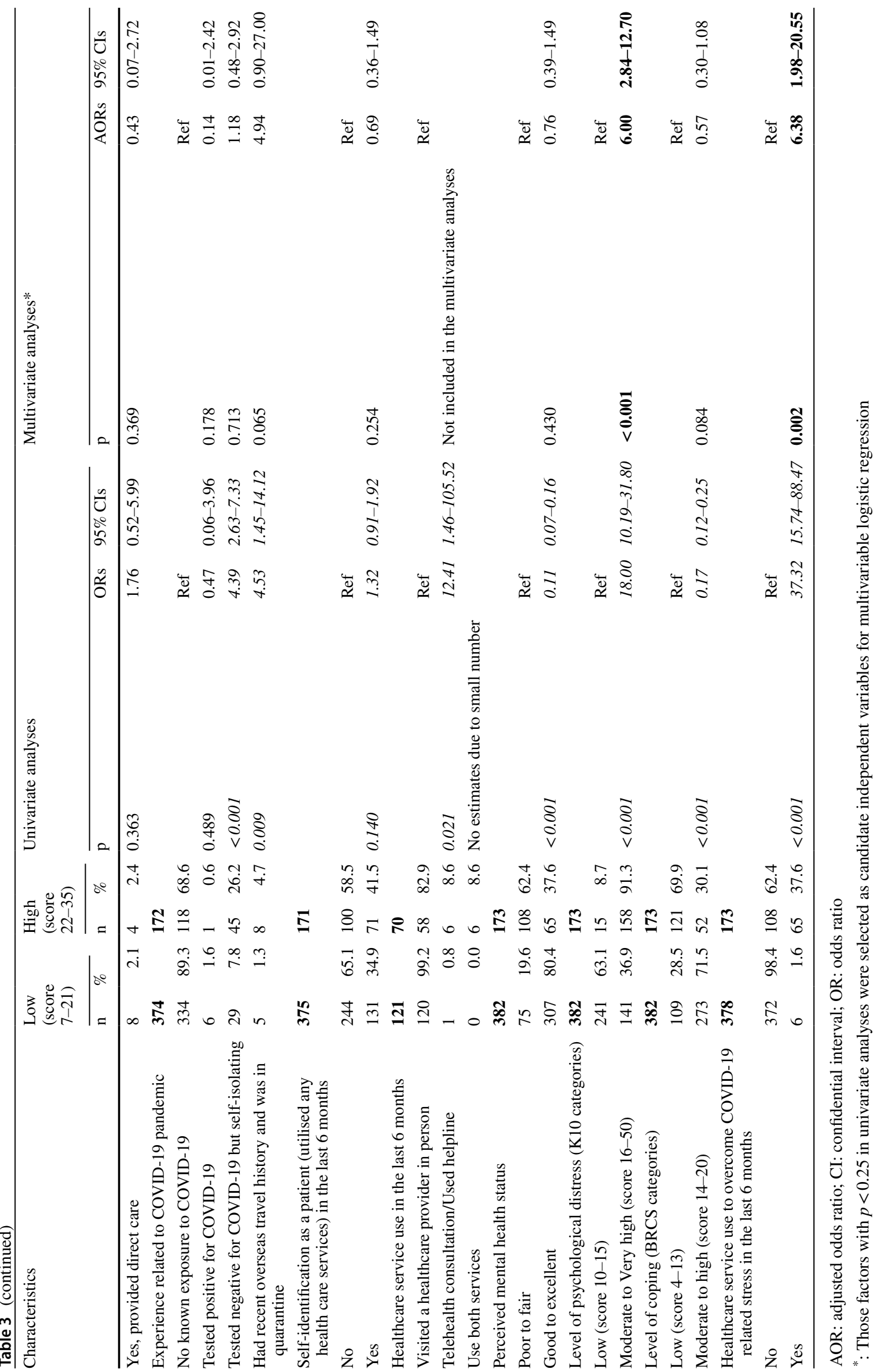


findings are somewhat consistent with the results of a similar Australian study (Rahman et al., 2020) using the same set of instruments. The Australian study showed that about twothirds (62.9\%) of Australian people experienced 'moderate to very high' levels of psychological distress. This finding was also similar to a recent study conducted in 194 cities in mainland China in which $53.8 \%$ of the respondents rated the psychological impact of the COVID-19 outbreak as moderate to severe (Wang et al., 2020). Both the Australian and this Hong Kong study report that about $30 \%$ of the participants had a high level of fear of the COVID-19 infection (Hong Kong: $31.2 \%$ and Australia: $31.9 \%$ ). However, the Australian participants demonstrated low resilient coping (97.3\%) versus a much lower percentage (about $40 \%$ ) in this Hong Kong study (Rahman et al., 2020). Resilience refers to the ability to withstand setbacks, adapt positively and rebound from adversity (Luthar \& Cicchetti, 2000). A tendency to effectively use cognitive appraisal skills in a flexible, committed approach to active problem solving despite stressful circumstances is described as "resilient coping behavior". People with high levels of resilient coping tend to believe in their abilities to address adverse circumstances and usually succeed at challenges (Sinclair \& Wallston, 2004). Moreover, those with higher resilience coping abilities showed less difficulty coping with the emotional challenges of the pandemic crisis (Killgore et al., 2020). Given that the Australian study (June 2020) was conducted six months before this study (December 2020 to Mid-January 2021), the participants in this study might have a better understanding of the COVID-19 infection and related information, the use of relevant preventive measures such as quarantine, social distancing and wearing masks, and successful stress management and coping strategies and experience sharing. As a result, people in Hong Kong during the later stage of the pandemic could show better coping through self-learning and resilience.

\section{Psychological Distress}

Living with family members, current alcohol consumption and a high level of fear of COVID-19 were associated with moderate to very high levels of psychological distress. Conversely, being older ( $30-59$ years, $\geq 60$ years), perceived good to excellent mental health, and moderate to high resilient coping were associated with low levels of psychological distress.

Living with family members was associated with moderate to very high levels of psychological distress in this study while the Australian study reported no association (Rahman et al, 2020). Hong Kong is well known as a city with highdensity housing and many are living in micro flats. Smaller residential size is associated with an increased risk of psychological distress among the general population (Wong et al., 2016). During this COVID-19 pandemic, the public has been advised to practice physical distancing, avoid crowded areas, and to work from home. Within a limited space in a small flat, living with family members may be equivalent to living with poorer personal space, leading to a higher level of psychological distress. For participants who have children, because children must study at home, working from home may mean heavier parental responsibilities, for example, dealing with parent-child relationships and monitoring children's study, therefore increasing their emotional burden (Wu et al., 2020).

Our study and Rahman et al. (2020) showed that current alcohol consumption was associated with moderate to very high levels of psychological distress. Increased alcohol consumption over the last six months was also related to a higher level of psychological distress and fear of COVID-19, and lower levels of coping strategies in univariate analyses. However, this variable was not included in the multivariate logistic regression due to limited responses. During the COVID-19 pandemic, adverse changes in health behaviours, mainly alcohol intake, were also associated with higher depression, anxiety and stress symptoms (Callinan et al., 2021; Stanton et al., 2020). Social isolation, quarantine, changes in employment status or uncertainty about the future, and any pandemic-related psychological distress may serve as significant triggers for increased alcohol intake (Ramalho, 2020; Stanton et al., 2020). Increased alcohol consumption might also be explained as a strategy to cope with perceived distress (Callinan et al., 2021; Stanton et al., 2020).

Being younger may be another potential risk factor for distress related to COVID-19 (Qiu et al., 2020; Yu et al., 2020). A nationwide study among 52,730 respondents in China showed that the young adult group, 18-30 years, reported the highest distress level during the pandemic (Qiu et al., 2020), which was consistent with the findings from our study and Australian (Rahman et al., 2020). The potential explanation might be that young participants are more likely to obtain pandemic-related information, including negative and inaccurate news from various social media and thus trigger stress (Qiu et al., 2020; Rahman et al., 2020). In addition, young people have the primary responsibility for social productivity and their family, and therefore bear more psychological pressure (Liu et al., 2020).

\section{Fear of COVID-19}

A high level of fear of COVID-19 was associated with the use of healthcare services to overcome COVID-19 related stress, moderate to a great deal of perceived stress due to change of employment conditions, self-identification as frontline or essential service worker, and moderate to very high psychological distress. Interestingly, our study 


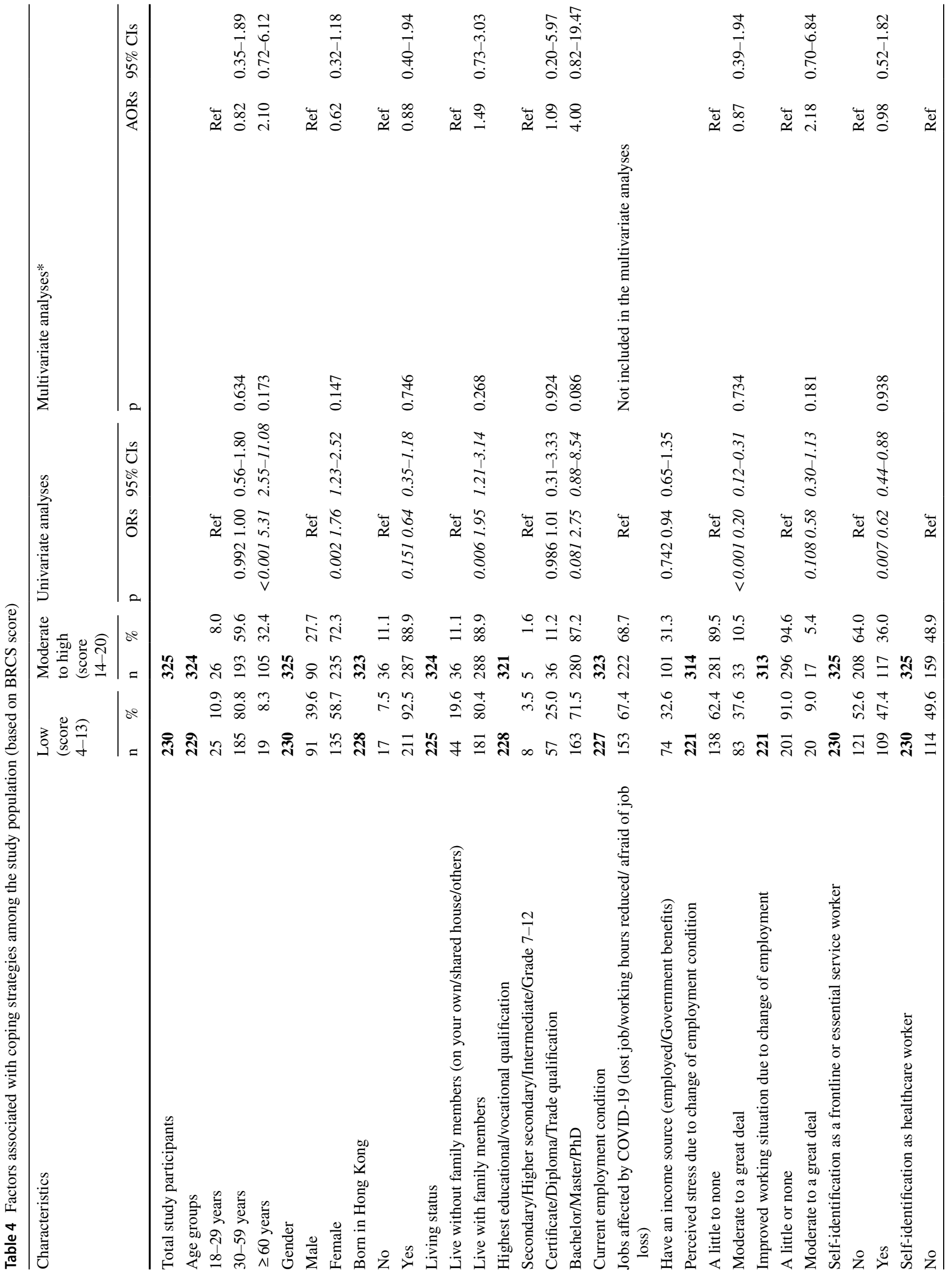




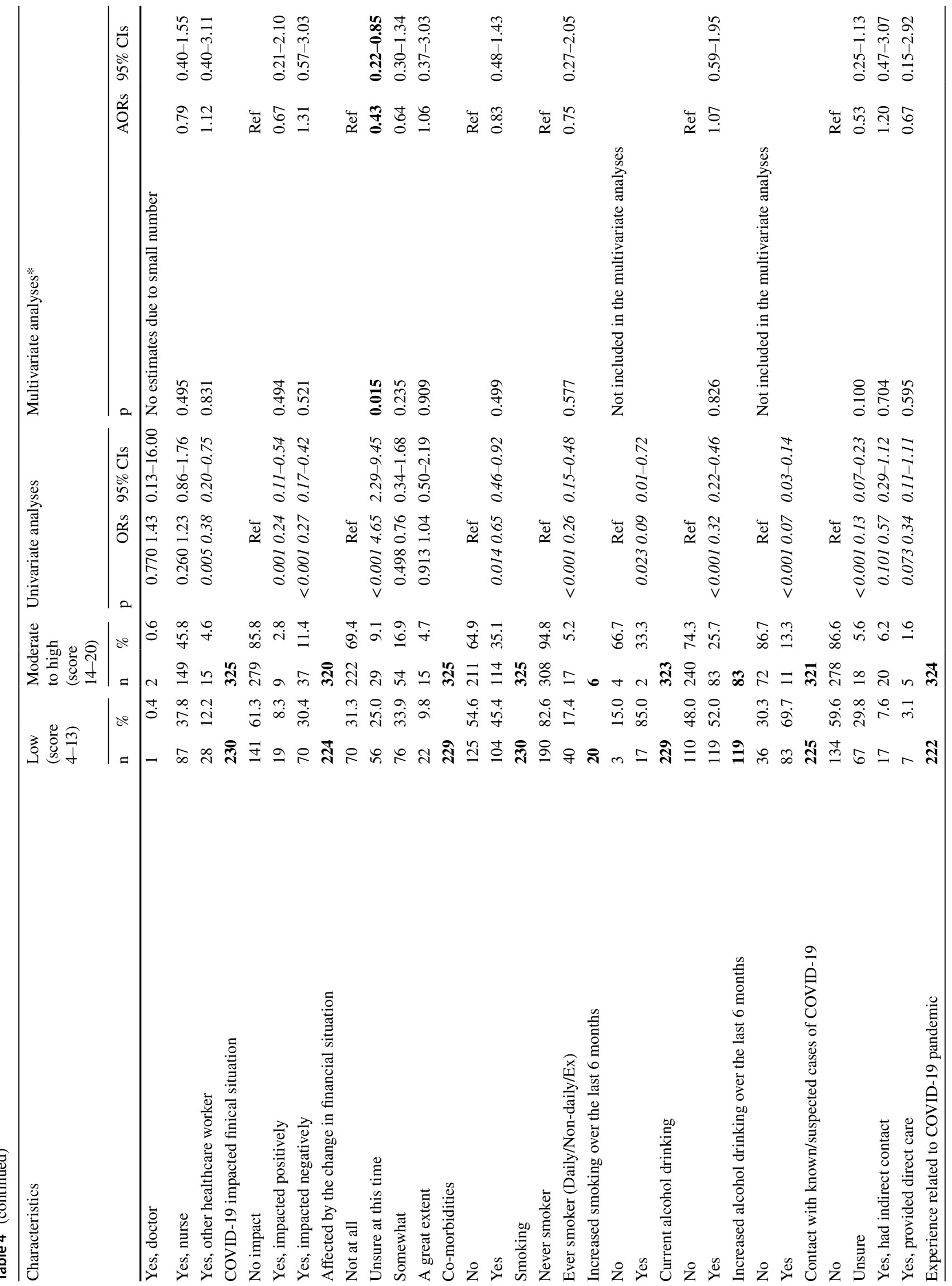




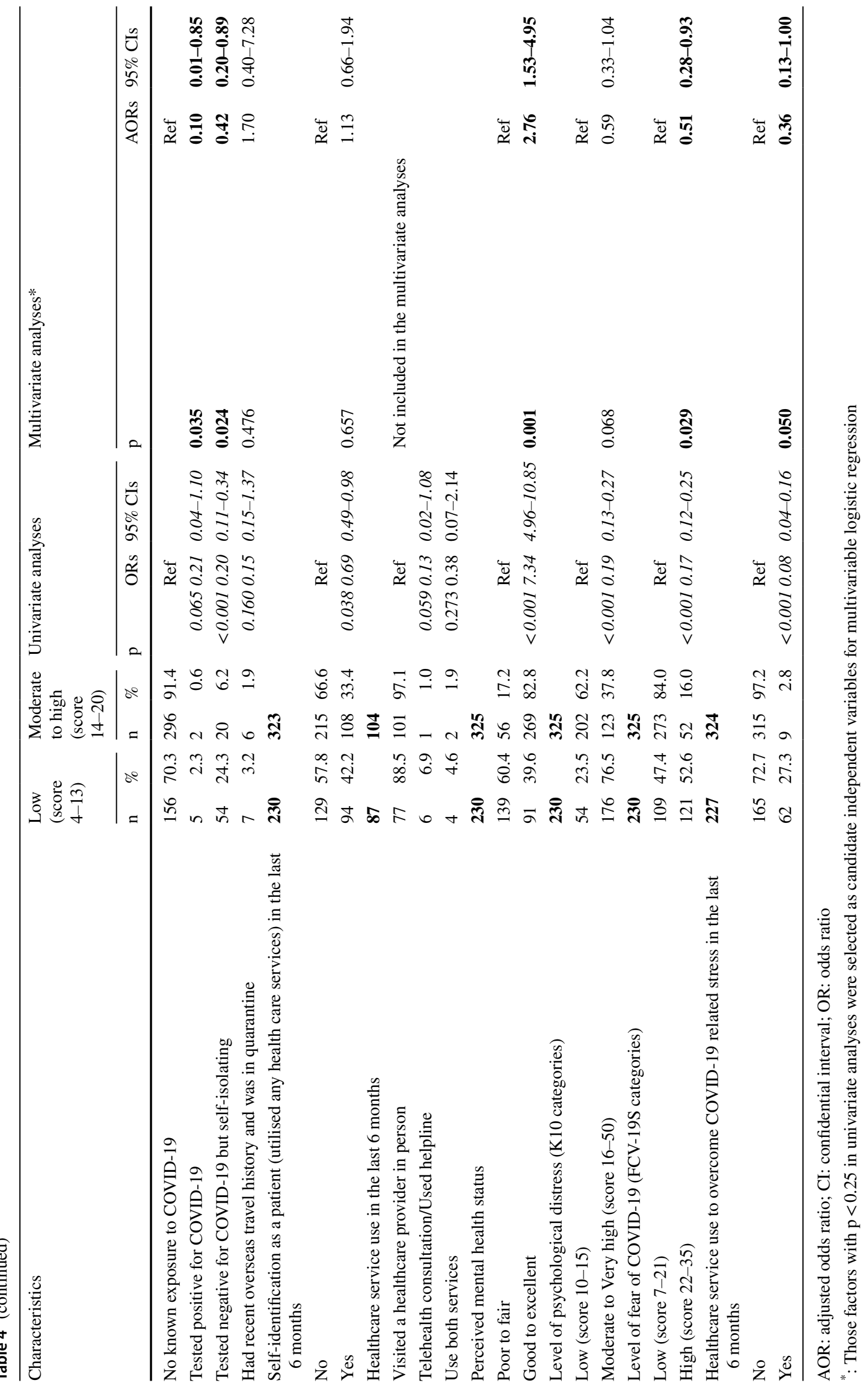


wwindicated that self-identification as a nurse was associated with a low level of fear of COVID-19.

Those who used healthcare services to overcome COVID19 related stress tended to report high levels of fear, probably because these participants had heightened self-awareness of their health. Moderate to a great deal of perceived stress due to a change in employment conditions was associated with higher levels of fear. The COVID-19 pandemic pushed millions of people into unemployment, underemployment and working poverty. According to the International Labour Organization (2020), almost 25 million jobs could be lost worldwide due to COVID-19. Among our participants, more than half $(68.2 \%)$ reported that their jobs were affected by COVID-19, such as job loss and reduced working hours. Approximately $20 \%$ of participants reported negatively impacted financial situation as a result of the pandemic. Therefore, it is important for the Hong Kong government to take effective strategies in time to reduce and improve the economic impacts.

Being a frontline or an essential service worker was associated with a high level of fear in this study. Such results are inconsistent with the Australian study, which reported that frontline or essential workers were less fearful than their counterparts (Rahman et al., 2020). However, our findings are in line with other studies which demonstrated a higher level of fear and psychological disorders among frontline healthcare workers than non-frontline workers (Cai et al., 2020; Lu et al., 2020). Likewise recent systematic reviews, have shown a considerable proportion of healthcare workers experienced various mental health problems during this outbreak (Magill et al., 2020; Pappa et al., 2020).

Compared with non-frontline workers or the general population, frontline healthcare workers may be more fearful and stressed. They may face a higher risk of exposure to COVID-19 as they need to spend more time in hospital wards, providing direct care to infected patients and some are responsible for virus detection as laboratory health workers. They may also be afraid of bringing the virus home to families and lack of ability to manage when facing critically ill patients. Due to their closer contact with patients, they may be more exposed to injury, suffering, and death (Cai et al., 2020; Lu et al., 2020; Pappa et al., 2020). In previous studies regarding frontline healthcare workers, nurses expressed a high level of fear of COVID-19 (Hu et al., 2020; Labrague \& de los Santos., 2021) and exhibited a higher prevalence of mental health problems than other healthcare workers (Pappa et al., 2020). However, in this study, the nurses who were mainly not frontline workers providing direct patient care reported a lower level of fear than nonhealthcare workers. Nonetheless, with more timely COVID19 information, knowledge about its prevention and control and availability of personal protective equipment, frontline healthcare workers could be less fearful than that in early stage of pandemic, and the general population who might not have adequate knowledge and resources for understanding and control of the COVID-19 pandemic (Zhang et al., 2020).

\section{Coping Strategies}

Good to excellent perceived mental health was associated with a moderate to high level of resilient coping. Change in financial situation, testing positive/negative for COVID19, high levels of fear of COVID-19 and using healthcare services to overcome COVID-19 related stress in the last six months were associated with low resilient coping. Interestingly, compared with the participants who had no known exposure to COVID-19, those who tested positive or negative for COVID-19 had lower levels of resilient coping. This could be explained where these participants, either being tested as positive or negative for COVID-19, having known exposure or possible contact with an infected person, and their perceived uncertainties about their own health status, might cause them to adopt maladaptive or avoidance coping responses, therefore lowering their resilience.

Our study found that the level of psychological distress was interrelated with the level of fear of COVID-19, indicating a positive mutual influence relationship. Moreover, in line with previous studies, our study found that resilience and psychological distress/fear are negatively associated (Killgore et al., 2020; Yasien et al., 2016), indicating that high resilience may help the public to adapt to the new norm during the pandemic, despite dealing with a fearful and stressful situation. Such psychological resilience-related interventions have also been applied in China during the pandemic and was shown to be to improve overall mental health among the general population (He et al., 2020).

\section{Limitations}

There are several limitations of this study. First, selection bias may occur due to using online platforms and only including those who were able to respond to the questionnaire in English. Therefore, the generalizability of the findings could be reduced by selection bias; and caution would be warranted when interpreting the study results. However, a remote data collection approach during the COVID-19 pandemic was deemed as a safe and effective way to collect survey data. Second, causal relationships could not be drawn due to the nature of cross-sectional design. In future, a prospective longitudinal study with larger-sized samples can be employed to examine the predictive relationships of wider varieties of important internal (personal) and external factors with psychological distress, fear and coping of different community groups during and after the pandemic. 


\section{Implications for Research and Practice}

Our study findings identified key factors associated with psychological distress, fear and coping. Local policymakers may consider necessary steps to reduce the effects of COVID-19. First, mental health services for different kinds of population, such as frontline healthcare workers, parents and young adults, can be delivered through eHealth such as video call consultations and hotlines to improve psychological well-being, and can be considered as precautionary measures for COVID-19 as well as physical distancing. Second, strategies aimed at adopting or maintaining heath behaviours should be promoted to avoid subsequent potential alcohol misuse and alcohol-related social harm and to address increase in psychological distress during the pandemic. Third, efforts by policymakers are needed to ensure proper, transparent, and timely dissemination of information related to COVID-19. The government, media and news organisations may need conjoint efforts to curb the spread of inaccurate media-fuelled infodemics that generate fear and panic. Resources for coping strategies during this pandemic are urgently needed to alleviate psychological distress. Despite more than half of the participants demonstrating moderate to high levels of resilient coping, $41.4 \%$ were demonstrated low resilient coping. Therefore, the development of series of resilience training activities is strongly recommended to reduce adverse mental health outcomes in a sudden public health epidemic.

\section{Conclusion}

The present study explored the key factors associated with psychological distress, fear of COVID-19 and coping strategies among the diverse population in Hong Kong. Mental health support strategies should be provided continuously to prevent the mental impact of the COVID-19 epidemic from turning into long-term illness.

Supplementary Information The online version contains supplementary material available at https://doi.org/10.1007/s12144-021-02338-7.

Funding The authors did not receive support from any organization for the submitted work.

Data Availability The datasets used and/or analysed during the current study are available from the corresponding author on reasonable request.

Code Availability Not applicable.

\section{Declarations}

Competing Interests The authors have no conflicts of interest to declare that are relevant to the content of this article.
Ethic approval and consent to participate This study was performed in line with the principles of the Declaration of Helsinki. Ethics approval was obtained from the Survey and Behavioural Research Ethics Committee at The Chinese University of Hong Kong (No. SBRE-20-172). Informed consent for participation in the study was obtained on the first page of the survey where study information was provided prior to participants completing the questionnaire.

Consent for publication Not applicable.

\section{References}

Ahorsu, D. K., Lin, C. Y., Imani, V., Saffari, M., Griffiths, M. D., \& Pakpour, A. H. (2020). The fear of COVID-19 scale: Development and initial validation. International Journal of Mental Health and Addiction, 1-9. Advance online publication. https://doi.org/10. 1007/s11469-020-00270-8

Ashraf, B. N. (2020). Economic impact of government interventions during the COVID-19 pandemic: International evidence from financial markets. Journal of Behavioral and Experimental Finance, 27, 100371. https://doi.org/10.1016/j.jbef.2020. 100371

Brooks, S. K., Webster, R. K., Smith, L. E., Woodland, L., Wessely, S., Greenberg, N., \& Rubin, G. J. (2020). The psychological impact of quarantine and how to reduce it: Rapid review of the evidence. The Lancet, 395(10227), 912-920. https://doi.org/10.1016/S01406736(20)30460-8

Cai, Q., Feng, H., Huang, J., Wang, M., Wang, Q., Lu, X., Xie, Y., Wang, X., Liu, Z., Hou, B., Ouyang, K., Pan, J., Li, Q., Fu, B., Deng, Y., \& Liu, Y. (2020). The mental health of frontline and non-frontline medical workers during the coronavirus disease 2019 (COVID-19) outbreak in China: A case-control study. Journal of Affective Disorders, 275, 210-215. https://doi.org/10. 1016/j.jad.2020.06.031

Callinan, S., Mojica-Perez, Y., Wright, C. J., Livingston, M., Kuntsche, S., Laslett, A. M., Room, R., \& Kuntsche, E. (2021). Purchasing, consumption, demographic and socioeconomic variables associated with shifts in alcohol consumption during the COVID-19 pandemic. Drug and Alcohol Review, 40(2), 183-191. https://doi. org/10.1111/dar.13200

Centre for Health Protection, Department of Health, The Government of the Hong Kong Special Administrative Region. (2021, May 21). Coronavirus disease 2019 [COVID-19]. Retrieved January 30, 2021, from https://www.chp.gov.hk/files/pdf/severe_respi ratory_disease_leaflet_en.pdf. Accessed 30 June 2021

Census and Statistics Department. The Government of the Hong Kong Special Administrative Region. (2021a, February 18). Table E016: Detailed statistical tables on labour force, employment, unemployment and underemployment (November 2020 to January 2021). Retrieved May 31, 2021, from https://www.chp. gov.hk/files/pdf/severe_respiratory_disease_leaflet_en.pdf

Census and Statistics Department, The Government of the Hong Kong Special Administrative Region. (2021b) Population estimates. Retreived May 31, 2021, from https://www.censtatd.gov.hk/en/ scode150.html

Chew, Q. H., Wei, K. C., Vasoo, S., Chua, H. C., \& Sim, K. (2020). Narrative synthesis of psychological and coping responses towards emerging infectious disease outbreaks in the general population: Practical considerations for the COVID-19 pandemic. Tropical Journal of Pharmaceutical Research, 61(7), 350-356. https://doi. org/10.11622/smedj.2020046 
Choi, E., Hui, B., \& Wan, E. (2020). Depression and anxiety in Hong Kong during COVID-19. International Journal of Environmental Research and Public Health, 17(10), 3740. https://doi.org/10. 3390/ijerph17103740

Fong, B. Y. F., Wong, M. C. S., Law, V. T. S., Lo, M. F., Ng, T. K. C., Yee, H. H. L., Leung, T. C. H., \& Ho, P. W. T. (2020a). Relationships between physical and social behavioural changes and the mental status of homebound residents in Hong Kong during the COVID-19 pandemic. International Journal of Environmental Research and Public Health, 17(18), 6653. https://doi.org/10. 3390/ijerph17186653

Fong, M. W., Gao, H., Wong, J. Y., Xiao, J., Shiu, E. Y., Ryu, S., \& Cowling, B. J. (2020b). Nonpharmaceutical measures for pandemic influenza in nonhealthcare settings social distancing measures. Emerging Infectious Diseases, 26(5), 976-984. https://doi. org/10.3201/eid2605.190995

Furukawa, T. A., Kessler, R. C., Slade, T., \& Andrews, G. (2003). The performance of the K6 and K10 screening scales for psychological distress in the Australian national survey of mental health and well-being. Psychological Medicine, 33(2), 357-362. https://doi. org/10.1017/S0033291702006700

Gan, X., Shi, Z., Chair, S. Y., Cao, X., \& Wang, Q. (2020). Willingness of Chinese nurses to practice in Hubei combating the coronavirus disease 2019 epidemic: A cross-sectional study. Journal of Advanced Nursing, 76(8), 2137-2150. https://doi.org/10.1111/ jan. 14434

He, Z., Chen, J., Pan, K., Yue, Y., Cheung, T., Yuan, Y., Du, N., Zhao, Y., Feng, Y., Zhou, D., Zhou, Y., Lu, F., Chen, Y., He, M., \& Xiang, Y. T. (2020). The development of the 'COVID-19 Psychological Resilience Model' and its efficacy during the COVID-19 pandemic in China. International Journal of Biological Sciences, 16(15), 2828-2834. https://doi.org/10.7150/ijbs.50127

Hu, D., Kong, Y., Li, W., Han, Q., Zhang, X., Zhu, L. X., Wan, S. W., Liu, Z., Shen, Q., Yang, J., He, H. G., \& Zhu, J. (2020). Frontline nurses' burnout, anxiety, depression, and fear statuses and their associated factors during the COVID-19 outbreak in Wuhan, China: A large-scale cross-sectional study. EClinicalMedicine, 24, 100424. https://doi.org/10.1016/j.eclinm.2020.100424

International Labour Organization. (2020, March 18). Almost 25 million jobs could be lost worldwide as a result of COVID-19, says ILO. Retrieved March 31, 2021, from https://www.ilo.org/global/ about-the-ilo/newsroom/news/WCMS_738742/lang--en/index. htm

Killgore, W. D., Taylor, E. C., Cloonan, S. A., \& Dailey, N. S. (2020). Psychological resilience during the COVID-19 lockdown. Psychiatry Research, 291, 113216. https://doi.org/10.1016/j.psych res.2020.113216

Kwok, K. O., Li, K. K., Chan, H. H. H., Yi, Y. Y., Tang, A., Wei, W. I., \& Wong, S. Y. S. (2020). Community responses during early phase of COVID-19 epidemic. Hong Kong. Emerging Infectious Diseases, 26(7), 1575-1579. https://doi.org/10.1101/2020.02.26. 20028217

Labrague, L. J., \& de los Santos, J. A. A. . (2021). Fear of COVID-19, psychological distress, work satisfaction and turnover intention among frontline nurses. Journal of Nursing Management, 29(3), 395-403. https://doi.org/10.1111/jonm.13168

Liu, X., Luo, W. T., Li, Y., Li, C. N., Hong, Z. S., Chen, H. L., Xiao, F., \& Xia, J. Y. (2020). Psychological status and behavior changes of the public during the COVID-19 epidemic in China. Infectious Diseases of Poverty, 9, 1-11. https://doi.org/10.1186/ s40249-020-00678-3

López, J., Perez-Rojo, G., Noriega, C., Carretero, I., Velasco, C., Martinez-Huertas, J. A., López-Frutos, P., \& Galarraga, L. (2020). Psychological well-being among older adults during the COVID-19 outbreak: A comparative study of the young-old and the old-old adults. International Psychogeriatrics, 32(11), 13651370. https://doi.org/10.1017/S1041610220000964

Lu, W., Wang, H., Lin, Y., \& Li, L. (2020). Psychological status of medical workforce during the COVID-19 pandemic: A crosssectional study. Psychiatry Research, 288, 112936. https://doi. org/10.1016/j.psychres.2020.112936

Luthar, S. S., \& Cicchetti, D. (2000). The construct of resilience: Implications for interventions and social policies. Development and Psychopathology, 12(4), 857-885. https://doi.org/10.1017/s0954 579400004156

Magill, E., Siegel, Z., \& Pike, K. M. (2020). The mental health of frontline health care providers during pandemics: A rapid review of the literature. Psychiatric Services, 71(12), 1260-1269. https:// doi.org/10.1176/appi.ps.202000274

Pappa, S., Ntella, V., Giannakas, T., Giannakoulis, V. G., Papoutsi, E., \& Katsaounou, P. (2020). Prevalence of depression, anxiety, and insomnia among healthcare workers during the COVID-19 pandemic: A systematic review and meta-analysis. Brain, Behavior, and Immunity, 88, 901-907. https://doi.org/10.1016/j.bbi.2020.05.026

Qiu, J., Shen, B., Zhao, M., Wang, Z., Xie, B., \& Xu, Y. (2020). A nationwide survey of psychological distress among Chinese people in the COVID-19 epidemic: Implications and policy recommendations. General Psychiatry, 33(2), e100213. https://doi.org/ 10.1136/gpsych-2020-100213

Ramalho, R. (2020). Alcohol consumption and alcohol-related problems during the COVID-19 pandemic: A narrative review. Australasian Psychiatry, 28(5), 524-526. https://doi.org/10.1177/ 1039856220943024

Rahman, M. A., Hoque, N., Alif, S. M., Salehin, M., Islam, S. M. S., Banik, B., Sharif, A., Nazim, N. B., Sultana, F., \& Cross, W. (2020). Factors associated with psychological distress, fear and coping strategies during the COVID-19 pandemic in Australia. Globalization and Health, 16(1), 1-15. https://doi.org/10.1186/ s12992-020-00624-w

Rahman, M. A., Salehin, M., Islam, S. M. S., Alif, S. M., Sultana, F., Sharif, A., Hoque, N., Nazim, N. B., \& Cross, W. M. (2021). Reliability of the tools used to examine psychological distress, fear of COVID-19 and coping amongst migrants and non-migrants in Australia. International Journal of Mental Health Nursing, 30, 745-756. https://doi.org/10.1111/inm.12845

Salari, N., Khazaei, H., Hosseinian Far, A., Khaledi-Paveh, B. Kazeminia, M., Mohammadi, M., Shohaimi, S., Daneshkhah, A., \& Eskandari, S. (2020). The prevalence of stress, anxiety and depression within front-line healthcare workers caring for COVID 19 patients: A systematic review and meta-regression. Human Resources for Health, 18(1), 100. https://doi.org/10.1186/ s12960-020-00544-1

Sansa, N. A. (2020). The Impact of the COVID-19 on the financial markets: Evidence from China and USA. Electronic Research Journal of Social Sciences and Humanities, 2(II), 29-39. https://doi.org/ 10.2139/ssrn.3567901

Sinclair, V. G., \& Wallston, K. A. (2004). The development and psychometric evaluation of the Brief Resilient Coping Scale. Assessment, 11(1), 94-101. https://doi.org/10.1177/1073191103258144

Stanton, R., To, Q. G., Khalesi, S., Williams, S. L., Alley, S. J., Thwaite, T. L., Fenning, A. S., \& Vandelanotte, C. (2020). Depression, anxiety and stress during COVID-19: Associations with changes in physical activity, sleep, tobacco and alcohol use in Australian adults. International Journal of Environmental Research and Public Health, 17(11), 4065. https://doi.org/10. 3390/ijerph17114065

The Government of the Hong Kong Special Administrative Region (2020, March). Hong Kong's multi-pronged response to COVID19 2020. Retrieved January 15, 2021, from https://www.hketo jakarta.gov.hk/doc/pdf/Factsheet_coronavirus_Mar_17_E.pdf 
The Government of the Hong Kong Special Administrative Region. (2021, June 21). Together, We Fight the Virus! Retrieved June 30, 2021, from https://www.coronavirus.gov.hk/eng/index.html

Tso, I. F., \& Park, S. (2020). Alarming levels of psychiatric symptoms and the role of loneliness during the COVID-19 epidemic: A case study of Hong Kong. Psychiatry Research, 293, 113423. https:// doi.org/10.1016/j.psychres.2020.113423

Tull, M. T., Edmonds, K. A., Scamaldo, K. M., Richmond, J. R., Rose, J. P., \& Gratz, K. L. (2020). Psychological outcomes associated with stay-at-home orders and the perceived impact of COVID-19 on daily life. Psychiatry Research, 289, 113098. https://doi.org/ 10.1016/j.psychres.2020.113098

Voßemer, J., Gebel, M., Täht, K., Unt, M., Högberg, B., \& Strandh, M. (2018). The effects of unemployment and insecure jobs on wellbeing and health: The moderating role of labor market policies. Social Indicators Research, 138(3), 1229-1257. https://doi.org/ 10.1007/s11205-017-1697-y

Wang, C., Pan, R., Wan, X., Tan, Y., Xu, L., Ho, C. S., \& Ho, R. C. (2020). Immediate psychological responses and associated factors during the initial stage of the 2019 coronavirus disease (COVID19) epidemic among the general population in China. International Journal of Environmental Research and Public Health, 17(5), 1729. https://doi.org/10.3390/ijerph17051729

Wong, C. S. M., Chan, W. C., Lam, L. C. W., Law, W. Y., Tang, W. Y., Wong, T. Y., \& Chen, E. Y. H. (2016). Living environment and psychological distress in the general population of Hong Kong. Procedia Environmental Sciences, 36, 78-81. https://doi.org/10. 1016/j.proenv.2016.09.016

Wong, S. Y. S., Zhang, D., Sit, R. W. S., Yip, B. H. K., Chung, R. Y., Wong, C. K. M., Chan, D. C. C., Sun, W., Kwok, K. O., \& Mercer, S. W. (2020). Impact of COVID-19 on loneliness, mental health, and health service utilisation: A prospective cohort study of older adults with multimorbidity in primary care. British Journal of General Practice, 70(700), e817-e824. https://doi.org/10.3399/ bjgp20X713021

World Health Organization (2020, March 11). WHO Director-General's opening remarks at the media briefing on COVID-19-11 March 2020. Retrieved May 15, 2021, from https://www.who.int/dg/ speeches/detail/who-director-general-s-opening-remarks-at-themedia-briefing-on-covid-19---11-march-2020
World Health Organization. (2021, June 21). WHO Coronavirus disease (COVID-19) Dashboard. Retrieved July 31, 2021, from https://covid19.who.int/

Wu, M., Xu, W., Yao, Y., Zhang, L., Guo, L., Fan, J., \& Chen, J. (2020). Mental health status of students' parents during COVID19 pandemic and its influence factors. General Psychiatry, 33(4), e100250. https://doi.org/10.1136/gpsych-2020-100250

Yao, R., \& Wu, W. (2021). Mental disorders associated with COVID19 related unemployment. Applied Research in Quality of Life. Published in Advance [5 May]. https://doi.org/10.1007/ s11482-021-09950-6

Yasien Abdul, S., Nasir, J., \& Shaheen, T. (2016). Relationship between psychological distress and resilience in rescue workers. Saudi Medical Journal, 37(7), 778-782. https://doi.org/10.15537/smj. 2016.7.15004

Yeung, N. C. Y., Huang, B., Lau, C. Y. K., \& Lau, J. T. F. (2020). Feeling anxious amid the COVID-19 pandemic: Psychosocial correlates of anxiety symptoms among Filipina domestic helpers in Hong Kong. International Journal of Environmental Research and Public Health, 17(21), 8102. https://doi.org/10.3390/ijerp h17218102

Yu, H., Li, M., Li, Z., Xiang, W., Yuan, Y., Liu, Y., Li, Z., \& Xiong, Z. (2020). Coping style, social support and psychological distress in the general Chinese population in the early stages of the COVID19 epidemic. BMC Psychiatry, 20(1), 1-11. https://doi.org/10. 1186/s12888-020-02826-3

Zhang, Y., Wang, C., Pan, W., Zheng, J., Gao, J., Huang, X., Cai, S., Zhai, Y., Latour, J. M., \& Zhu, C. (2020). Stress, burnout, and coping strategies of frontline nurses during the COVID-19 epidemic in Wuhan and Shanghai. China. Frontiers in Psychiatry, 11, 565520. https://doi.org/10.3389/fpsyt.2020.565520

Zhao, S. Z., Wong, J. Y. H., Luk, T. T., Wai, A. K. C., Lam, T. H., \& Wang, M. P. (2020). Mental health crisis under COVID-19 pandemic in Hong Kong, China. International Journal of Infectious Diseases, 100, 431-433. https://doi.org/10.1016/j.ijid.2020. 09.030

Publisher's Note Springer Nature remains neutral with regard to jurisdictional claims in published maps and institutional affiliations. 